\title{
Teleportation transfers only speakable quantum information
}

\author{
Giulio Chiribella ${ }^{1}$, Vittorio Giovannetti ${ }^{2}$, Lorenzo Maccone ${ }^{3}$, Paolo Perinotti ${ }^{3}$ \\ ${ }^{1}$ Center for Quantum Information, Institute for Interdisciplinary \\ Information Sciences, Tsinghua University, Beijing 100084, China. \\ 2 NEST, Scuola Normale Superiore \& CNR-INFM, Piazza dei Cavalieri 7, I-56126, Pisa, Italy. \\ ${ }^{3}$ QUIT group, Dip. Fisica, Università di Pavia and INFN, via A. Bassi 6, I-27100 Pavia, Italy.
}

\begin{abstract}
We show that a quantum clock cannot be teleported without prior synchronization between sender and receiver: every protocol using a finite amount of entanglement and an arbitrary number of rounds of classical communication will necessarily introduce an error in the teleported state of the clock. Nevertheless, we show that entanglement can be used to achieve synchronization with precision higher than any classical correlation allows, and we give the optimized strategy for this task. The same results hold also for arbitrary continuous quantum reference frames, which encode general unspeakable information,- - information that cannot be encoded into a number, but instead requires a specific physical support, like a clock or a gyroscope, to be conveyed.

PACS numbers: 03.67.-a,03.67.Hk,03.65.Ud,91.10.Ws
\end{abstract}

Speakable Information (SI) refers to those messages, such as the content of this paper, for which the means of encoding is not important and which can be represented as a string of bits. On the contrary, Unspeakable Information (UI) refers to those messages, such as the specification of a direction in space or a time reference, which require a specific physical system to be transferred [1] 3 . UI can be mapped into SI in the presence of a shared Reference Frame (RF): e.g. the sender of the message (Alice) can specify a direction in space by transferring to the receiver (Bob) a set of coordinates with respect to their shared Cartesian frame. In quantum mechanics the difference between SI and UI can be highlighted as follows: Consider a quantum system $S$, whose preparation is described by Alice as the quantum state $|\psi\rangle=\sum_{n} \alpha_{n}|n\rangle$, where $\{|n\rangle\}_{n=1}^{d}$ is Alice's canonical basis. Assuming that Alice knows the expansion coefficients $\left\{\alpha_{n}\right\}_{n=1}^{d}$, we can identify those quantities as the SI content of the state (this is her "description" of the system and she can transfer it to Bob by simply exchanging bits), whereas we can identify Alice's basis $\{|n\rangle\}_{n=1}^{d}$ with the UI content of $|\psi\rangle$. To transfer $|\psi\rangle$ Alice needs either to send the system itself, or to make sure that she shares with Bob the same canonical basis, which plays the role of the shared RF. If Alice has no information on $|\psi\rangle$, but she shares entanglement with Bob, she may use quantum teleportation [4] to transfer the state to him by only sending bits. Does this imply that shared entanglement can compensate for the absence of a shared RF?

In this Rapid Communication we answer the question with a general no-go theorem: in the absence of a prior shared RF, UI cannot be reliably teleported, the only exception being RFs associated to finite symmetry groups. For example, in the lack of common time reference between Alice and Bob, a teleported clock cannot remain synchronized with the sender's clock. Previous work [3, 目 7] has shown that the lack of a shared RF can reduce the amount of usable entanglement that the parties share, or may prevent to establish an isomorphism between Alice's and Bob's Hilbert spaces [8]. However, this per se does not imply the impossibility of devising suitable teleportation protocols, e.g. using invariant entangled states [9]. Our general no-go theorem establishes that these protocols can transfer only SI, whereas UI teleportation is impossible.

Even though perfect UI teleportation is impossible, entanglement is still a useful resource even in the absence of shared RF. In particular, approximate UI teleportation is possible with vanishing error in the limit of infinite entangled resources. This can be achieved by twostep protocols where some entanglement is used to establish an approximate shared RF, and then one uses ordinary teleportation to transfer UI in the same way as one would do for SI. In this scenario, the crucial step is the optimal extraction of a shared RF from a bipartite state. Non-optimal protocols to convert prior entanglement into shared RF were previously proposed in 10, 11] and analyzed in [12]. Here we present the optimal protocol, which, in contrast to prior protocols, displays a quantum-metrology enhancement 13]. Our result allows us to explicitly quantify the usefulness of a pure bipartite state for clock synchronization, through a family of "measures of frameness" 14, 15] that are monotone under deterministic local operations and classical communication (LOCC) in the lack of a shared RF. Differing from other recent works in the RF literature [16, 17], which focus on the single-party scenario, this result starts the quantification of the resourcefulness of quantum RFs in the bipartite setting.

\section{IMPOSSIBILITY OF UI-TELEPORTATION}

We start by considering the case in which Alice and Bob lack of a common phase reference. This implies that the RFs of Alice and Bob differ by an unknown (but fixed) element of the group $U(1)$, corresponding to the phase mismatch $\varphi$. Given a generic quantum system $S$, let us indicate with $G_{S}$ the generator of the representation of the phase shifts on $S$ (here $G_{S}$ can be any operator 
with integer eigenvalues). Define also $\left|\psi^{A}\right\rangle$ and $\left|\psi^{B}\right\rangle$ to be the states of $S$ associated to the same $S I$ description produced by Alice and Bob, respectively (i.e. states with same expansion coefficients on the eigenvectors of $G_{S}$ ). Due to the lack of a common phase reference between Alice's and Bob's eigenvectors, those two states differ and are related by the unitary transformation $U_{\varphi}=e^{-i \varphi G_{S}}$,

$$
\left|\psi^{B}\right\rangle=U_{\varphi}\left|\psi^{A}\right\rangle
$$

Conversely, denoting by $\alpha_{n}^{(A)}$ and $\alpha_{n}^{(B)}$ the expansion coefficients of the same state $|\psi\rangle$ of $S$ with respect to the eigenvectors of $G_{S}$ in Alice's and Bob's canonical basis, they will be related by the transformation $\alpha_{n}^{(B)}=$ $\alpha_{n}^{(A)} \exp \left[i \varphi g_{n}\right], g_{n} \in \mathbb{Z}$ being the $n$-th eigenvalue of $G_{S}$.

Let $\left|\psi_{0}^{A}\right\rangle$ be an input state with nontrivial UI content, that is, a state such that $U_{\varphi}\left|\psi_{0}^{A}\right\rangle \neq\left|\psi_{0}^{A}\right\rangle$, and consider the state $\left|\psi_{\theta}^{A}\right\rangle:=U_{\theta}\left|\psi_{0}^{A}\right\rangle$ where $\theta$ is an arbitrary phase shift. The teleportation of $U I$ is any protocol where Alice transfers $\left|\psi_{\theta}^{A}\right\rangle$ to Bob using only a prior shared entangled state and classical communication. In contrast, the teleportation of $S I$ [9] is any protocol that produces as output the state $\left|\psi_{\theta}^{B}\right\rangle$, that is, the state that in Bob's RF has the same coefficients of $\left|\psi_{\theta}^{A}\right\rangle$ in Alice's. In both cases, depending on the degrees of freedom involved, establishing the entangled state for teleportation may or may not require prior transfer of UI.

We now prove that UI teleportation is impossible for any finite entangled resource and for arbitrarily many rounds of classical communication. Some intuition on the impossibility can be gained by considering the standard teleportation protocol [ 4 : here, in order to be able to retrieve Alice's state Bob has to correct the error introduced by Alice's Bell measurement. However, if their reference frames differ, then Bob's operations will sometimes differ from the required corrections and thus will not be able to undo the error. Hence, the standard teleportation protocol cannot be used to perfectly transfer UI. Our no-go theorem extends this impossibility to arbitrary protocols.

Proof: Define the Hilbert spaces $\mathcal{H}_{S}$ for the system to be teleported, and $\mathcal{H}_{A}, \mathcal{H}_{B}$ for the entangled state $|E\rangle \in \mathcal{H}_{A} \otimes \mathcal{H}_{B}$, here expressed in Alice's description. Adopting Alice's description, Bob's quantum operations $\mathcal{B}$ from states on $\mathcal{H}_{B}$ to states on $\mathcal{H}_{S}$ will be described as $\mathcal{U}_{\varphi} \mathcal{B} \mathcal{W}_{\varphi}^{\dagger}$, where $\mathcal{U}_{\varphi}(\rho)=U_{\varphi} \rho U_{\varphi}^{\dagger}$ and $\mathcal{W}_{\varphi}^{\dagger}(\rho)=W_{\varphi}^{\dagger} \rho W_{\varphi}$, $W_{\varphi}=e^{-i \varphi G_{B}}$ being the unitary transformation implementing the phase shift $\varphi$ on $\mathcal{H}_{B}$ - see Eq. (11). Since teleportation uses only LOCC resources it will be described by a separable quantum channel of the form $\mathcal{C}=\sum_{k} \mathcal{A}_{k} \otimes \mathcal{U}_{\varphi} \mathcal{B}_{k} \mathcal{W}_{\varphi}^{\dagger}$, where $\mathcal{A}_{k}$ are the operations performed by Alice (they annihilate states on $\mathcal{H}_{S} \otimes \mathcal{H}_{A}$ ), $\mathcal{B}_{k}$ are the operations performed by Bob (they send states on $\mathcal{H}_{B}$ to states on $\mathcal{H}_{S}$ ), while the index $k$ keeps track of all the outcomes and of the classical communication exchanged during the protocol. The condition of perfect
UI-teleportation is then

$$
\sum_{k}\left(\mathcal{A}_{k} \otimes \mathcal{U}_{\varphi} \mathcal{B}_{k} \mathcal{W}_{\varphi}^{\dagger}\right)\left(\left|\psi_{\theta}^{A}\right\rangle\left\langle\psi_{\theta}^{A}|\otimes| E\right\rangle\langle E|\right)=\left|\psi_{\theta}^{A}\right\rangle\left\langle\psi_{\theta}^{A}\right|
$$

for every $\varphi$ and $\theta$. Without loss of generality we can assume each $\mathcal{B}_{k}$ to be a covariant quantum operation, satisfying $\mathcal{U}_{\varphi} \mathcal{B}_{k} \mathcal{W}_{\varphi}^{\dagger}=\mathcal{B}_{k}$ for every $\varphi$ [18]. Hence, $\varphi$ disappears from Eq. (2). Moreover, applying $\mathcal{U}_{\theta}^{\dagger}$ on both sides of Eq. (2), using covariance, and averaging over $\theta$, we obtain $\left|\psi_{0}^{A}\right\rangle\left\langle\psi_{0}^{A}\right|=\mathcal{C}(\sigma)$, where

$$
\sigma:=\int_{0}^{2 \pi} \frac{d \theta}{2 \pi}\left(\mathcal{U}_{\theta} \otimes \mathcal{I}_{A} \otimes \mathcal{W}_{\theta}^{\dagger}\right)\left(\left|\psi_{0}^{A}\right\rangle\left\langle\psi_{0}^{A}|\otimes| E\right\rangle\langle E|\right) .
$$

Computing the integral we then get $\sigma=\sum_{l} \sigma_{l}$, where $\sigma_{l}:=\Pi_{l}\left(\left|\psi_{0}^{A}\right\rangle\left\langle\psi_{0}^{A}|\otimes| E\right\rangle\langle E|\right) \Pi_{l}$ and $\Pi_{l}$ is the projector on the eigenspace of the operator $G_{-}:=G_{S} \otimes \mathbb{1}_{A} \otimes$ $\mathbb{1}_{B}-\mathbb{1}_{S} \otimes \mathbb{1}_{A} \otimes G_{B}$ corresponding to the eigenvalue $l$. Now, the condition $\left|\psi_{0}^{A}\right\rangle\left\langle\psi_{0}^{A}\right|=\mathcal{C}(\sigma)$ implies $\mathcal{C}\left(\sigma_{l}\right) \propto$ $\left|\psi_{0}^{A}\right\rangle\left\langle\psi_{0}^{A}\right|$ for every $l$. Let us denote by $P_{m}\left(Q_{n}\right)$ the projector on the eigenspace of $G_{S}\left(G_{B}\right)$ with eigenvalue $m(n)$, and by $m_{\max }\left(n_{\min }\right)$ the maximum $m$ (minimum $n)$ such that $P_{m}\left|\psi_{0}^{(A)}\right\rangle \neq 0\left(\left(I_{A} \otimes Q_{n}\right)|E\rangle \neq 0\right)$. Defining $l_{\max }:=m_{\max }-n_{\min }$ we then have $\sigma_{l_{\max }}=\left(P_{m_{\max }} \otimes I_{A} \otimes\right.$ $\left.Q_{n_{\min }}\right) \sigma_{0}\left(P_{m_{\max }} \otimes I_{A} \otimes Q_{n_{\min }}\right)$. This equation implies that $\sigma_{l_{\max }}$ is invariant under phase shifts on system $\mathcal{H}_{B}$. As a consequence, also $\mathcal{C}\left(\sigma_{l_{\text {max }}}\right)$ must be invariant under phase shifts: $\mathcal{U}_{\varphi} \mathcal{C}\left(\sigma_{l_{\max }}\right)=\sum_{k}\left(\mathcal{A}_{k} \otimes \mathcal{U}_{\varphi} \mathcal{B}_{k}\right)\left(\sigma_{l_{\max }}\right)=$ $\sum_{k}\left(\mathcal{A}_{k} \otimes \mathcal{B}_{k} \mathcal{W}_{\varphi}\right)\left(\sigma_{l_{\max }}\right)=\mathcal{C}\left(\sigma_{l_{\max }}\right), \forall \varphi \in[0,2 \pi]$. Since $\mathcal{C}\left(\sigma_{l_{\max }}\right)$ is invariant and $\mathcal{C}\left(\sigma_{l_{\max }}\right) \propto\left|\psi_{0}^{A}\right\rangle\left\langle\psi_{0}^{A}\right|$, we proved that $\left|\psi_{0}^{A}\right\rangle\left\langle\psi_{0}^{A}\right|$ must be invariant, in contradiction with the hypothesis that $\left|\psi_{0}^{A}\right\rangle$ has a nontrivial UI content.

Our no-go theorem differs from previous results 3, 5, 8, 9] in two important respects: i) the result is stronger because it states the impossibility of teleportation even for a restricted set of states; ii) The reason why UIteleportation is impossible is not that the entangled resource is degraded by the lack of a shared phase reference between Alice and Bob [5, 12]: in our setting the state $|E\rangle$ could be invariant under global changes of phase, and the no-go theorem would still hold. Our proof, derived for RF mismatches associated with transformations $U(1)$, can be immediately generalized to any continuous compact Lie group $\mathbf{G}$, as any $\mathbf{G}$ contains at least one subgroup that is isomorphic to $U(1)$. In contrast, our derivation does not apply to the case in which the lack of shared RF is associated to finite group transformations (e.g. chirality). In this case, one can always achieve perfect teleportation with a two-step protocol where a shared RF is established before using conventional teleportation 4, 19. To do so, Alice and Bob can use the state $|E\rangle \propto \sum_{h \in \mathbf{G}}|h\rangle|h\rangle$, where $\{|h\rangle, h \in \mathbf{G}\}$ are orthonormal vectors transforming as $U_{g}|h\rangle=|g h\rangle$ : if Alice and Bob measure on this basis, their outcomes will be $h$ and $g h$, respectively, and from this information they can infer the mismatch $g$.

A remarkable consequence of our no-go theorem is the impossibility to teleport a quantum clock without prior 
synchronization. If Alice and Bob are not synchronized, the times $t^{A}$ and $t^{B}$ each of them attributes to the same event are related by $t^{B}=t^{A}+\delta t$, where $\delta t$ is the offset between their clocks. A quantum clock undergoes a periodic evolution $U_{t}=e^{-i t H / \hbar}$, where $H=\sum_{n=0}^{N} n E_{0}|n\rangle\langle n|$ is the Hamiltonian. The state of the clock at time $t^{A}$, given by $\left|\psi_{t^{A}-t_{0}^{A}}\right\rangle=U_{t^{A}-t_{0}^{A}}|\psi\rangle:=U_{\tau}|\psi\rangle$, carries information about the time $\tau$ elapsed since the initial time $t_{0}^{A}$ when the evolution started. The goal of clock teleportation is to allow Bob to measure the time $t_{0}^{B}$ when the beginning of the oscillations took place according to his time reference. This means that, if the duration of the protocol is $T$, Bob's output state must be $\left|\psi_{\tau+T}\right\rangle$. The duration $T$ is unknown (otherwise Alice and Bob could easily synchronize their clocks), and this translates into a lack of a shared phase reference: Indeed, consider a single-round protocol, where $T$ is the time elapsed between Alice's and Bob's actions. During that time, the system at Bob's side will evolve according to the unitary $W_{T}=e^{-i H_{B} T / \hbar}$, where $H_{B}$ is the free Hamiltonian. The condition for perfect teleportation is then $\sum_{k}\left(\mathcal{A}_{k} \otimes \mathcal{B}_{k} \mathcal{W}_{T}\right)\left(\left|\psi_{\tau}\right\rangle\left\langle\psi_{\tau}|\otimes| E\right\rangle\langle E|\right)=\mathcal{U}_{T}\left(\left|\psi_{\tau}\right\rangle\left\langle\psi_{\tau}\right|\right)$, which must hold for every $\tau$ and $T$. Since this equation is equivalent to Eq. (2), the impossibility proof for UI teleportation carries over and teleportation of the quantum clock is impossible. The above argument immediately extends to multi-round protocols because only the first interval $T$ is unknown, while the subsequent ones at which Bob applies successive transformations can be measured by him locally, and accounted for with his known Hamiltonian: successive iterations do not add anything to the protocol.

We have seen that teleportation of UI is impossible. Teleportation of SI is instead possible. The condition of perfect SI-teleportation is

$$
\sum_{k}\left(\mathcal{A}_{k} \otimes \mathcal{U}_{\varphi} \mathcal{B}_{k} \mathcal{W}_{\varphi}^{\dagger}\right)\left(\left|\psi_{\theta}^{A}\right\rangle\left\langle\psi_{\theta}^{A}|\otimes| E\right\rangle\langle E|\right)=\left|\psi_{\theta}^{B}\right\rangle\left\langle\psi_{\theta}^{B}\right|,
$$

for every $\varphi$ and $\theta$. With respect to Eq. (2), the rhs of Eq. (3) acquires an extra rotation $U_{\varphi}$, which cancels the one on the lhs. Choosing system $B$ to be invariant under phase shifts we have $W_{\varphi}=I_{B}$ for every $\varphi$ and the equation becomes $\sum_{k}\left(\mathcal{A}_{k} \otimes \mathcal{B}_{k}\right)\left(\left|\psi_{\theta}^{A}\right\rangle\left\langle\psi_{\theta}^{A}|\otimes| E\right\rangle\langle E|\right)=$ $\left|\psi_{\theta}^{A}\right\rangle\left\langle\psi_{\theta}^{A}\right|$ for every $\theta$. This condition can be achieved by using an ordinary teleportation protocol: the only constraint is that system $B$ has to be an invariant degree of freedom. In the context of synchronization, the basic ideas behind those schemes is that Alice transfers the quantum state her clock to some energy-degenerate degrees of freedom that do not evolve (this can be done with a local teleportation in her lab). The state of these degrees of freedom can be teleported to Bob even in the lack of synchronization, using the protocols presented of Refs. [3, 9]. Bob can then locally transfer the quantum information he received on his quantum clock (through a local teleportation in his lab). This gives back a ticking clock, which however is no longer synchronized with
Alice's.

\section{OPTIMAL REMOTE SYNCHRONIZATION}

Although perfect UI-teleportation is impossible for any finite entangled resource, approximate UI-teleportation is achievable with arbitrary precision. E.g., one can use a two-step protocol where Alice and Bob use part of the entangled resource to establish a shared RF. Here we focus on the latter task and give the optimal strategy to find the estimate of the phase mismatch $\varphi$ using a given entangled state. This result optimizes quantum clock synchronization based on prior entanglement [10, 11.

Suppose that the resource state $|E\rangle \in \mathcal{H}_{A} \otimes \mathcal{H}_{B}$ is invariant under global phase-shifts $V_{\varphi} \otimes W_{\varphi}$ and that the generators of $V_{\varphi}$ and $W_{\varphi}$ can be written as $G_{A} \equiv$ $\sum_{n=0}^{N_{A}-1} n P_{n}$ and $G_{B} \equiv \sum_{n=0}^{N_{B}-1} n Q_{n}$. Equivalently, $|E\rangle$ is an eigenstate of the sum $G_{A}+G_{B}$ for some eigenvalue $N$, namely

$$
|E\rangle \equiv \sum_{n=0}^{N} e_{n}\left|E_{n}\right\rangle, \quad\left|E_{n}\right\rangle \equiv \frac{\left(P_{N-n} \otimes Q_{n}\right)|E\rangle}{\|\left(P_{N-n} \otimes Q_{n}\right)|E\rangle \|}
$$

The synchronization protocol is described by an LOCC POVM, whose outcome is the estimate $\hat{\varphi}$. Adopting Alice's description (11), the POVM elements can be expressed as $M_{\hat{\varphi}}^{(\varphi)}=\left(\mathbb{1}_{A} \otimes W_{\varphi}\right) M_{\hat{\varphi}}^{(0)}\left(\mathbb{1}_{A} \otimes W_{\varphi}^{\dagger}\right)$, and obey the normalization condition $\int \frac{\mathrm{d} \hat{\varphi}}{2 \pi} M_{\hat{\varphi}}^{(\varphi)}=\mathbb{1}_{A} \otimes \mathbb{1}_{B}$, for all $\varphi$. A protocol is optimal if it minimizes the average cost

$$
\langle c\rangle=\int \frac{\mathrm{d} \varphi}{2 \pi} \int \frac{\mathrm{d} \hat{\varphi}}{2 \pi} c(\hat{\varphi}-\varphi)\left\langle E\left|M_{\hat{\varphi}}^{(\varphi)}\right| E\right\rangle,
$$

where $c(\hat{\varphi}-\varphi)$ is a cost function dependent on the difference between the true value $\varphi$ and the estimate $\hat{\varphi}$. Note that the optimal $M_{\hat{\varphi}}^{(\varphi)}$ can always be chosen of the form $M_{\hat{\varphi}}^{(\varphi)}=\left(\mathbb{1}_{A} \otimes W_{\hat{\varphi}-\varphi}\right) \Xi\left(\mathbb{1}_{A} \otimes W_{\hat{\varphi}-\varphi}^{\dagger}\right)$, with $\Xi$ positive operator on $\mathcal{H}_{A} \otimes \mathcal{H}_{B}$. Indeed, suppose that $M_{\hat{\varphi}}^{(\varphi)}$ is not of this form, then we can replace it with its average $\widetilde{M}_{\hat{\varphi}}^{(\varphi)} \equiv \int \frac{\mathrm{d} \theta}{2 \pi} M_{\hat{\varphi}+\theta}^{(\varphi+\theta)}$ without modifying the average cost. The new POVM $\widetilde{M}_{\hat{\varphi}}^{(\varphi)}$ still describes a LOCC protocol: Alice and Bob can achieve it by randomly shifting Bob's phase by $\theta$, measuring the old $\operatorname{POVM} M_{\hat{\varphi}}^{(\varphi)}$, and shifting the estimate $\hat{\varphi}$ back by $\theta$.

With this observation, the problem is reduced to the estimation of the local phase shift $\left(\mathbb{1}_{A} \otimes W_{\varphi}\right)$ on the input state $|E\rangle$. The optimal solution of this problem is known [20, 21] for cost functions of the form $c(\varphi)=\sum_{q=1}^{\infty} c_{q} \cos (q \varphi)$ with $c_{q} \leq 0$ for $q \neq 0$ : the minimum cost over all joint POVMs is given by $\langle c\rangle_{\min }^{\text {joint }}=$ $\sum_{q} c_{q} \sum_{n}\left|e_{n} e_{n+q}\right|$, where $\left\{e_{n}\right\}$ are the expansion coefficients in Eq. (4). Now we show that the minimum cost is achieved by a one-way LOCC protocol. For simplicity, let us start from the case of nondegenerate energy levels, for which $P_{n}=|n\rangle_{A}\langle n|$ and $Q_{n}=|n\rangle_{B}\langle n|$. In this case, 
Alice can measure on the Fourier basis

$$
\left|a_{k}\right\rangle_{A}=\frac{1}{\sqrt{N_{A}}} \sum_{n=0}^{N_{A}-1} \omega^{k n}|n\rangle_{A},
$$

where $\omega:=e^{2 \pi i / N_{A}}$. For outcome $k$, the state of Bob's system is $W_{\varphi}\left|\psi_{k}\right\rangle$, with $\left|\psi_{k}\right\rangle=\sum_{n=0}^{S} e_{n} \omega^{k n}|n\rangle_{B}$. Then Bob can perform the optimal phase estimation on this state, achieving the minimum cost. In general, Alice and Bob's $n$-th energy levels have degeneration $d_{n, A}$ and $d_{n, B}$. The state $\left|E_{n}\right\rangle$ can then be entangled, with Schmidt form $\left|E_{n}\right\rangle=\sum_{l_{n}=1}^{r_{n}} \lambda_{n, l_{n}}\left|N-n, l_{n}\right\rangle_{A}\left|n, l_{n}\right\rangle_{B}$. Then Alice can measure the Fourier basis

$\left|a_{k,\left\{j_{n}\right\}}\right\rangle_{A}=\frac{1}{\sqrt{N_{A}}} \sum_{n=0}^{N_{A}-1} \omega^{k n}\left(\frac{1}{\sqrt{d_{n, A}}} \sum_{l_{n}=1}^{d_{n, A}} v^{j_{n} l_{n}}\left|n, l_{n}\right\rangle_{A}\right)$,

with $\omega:=e^{\frac{2 \pi i}{N_{A}}}$ and $v:=e^{\frac{2 \pi i}{d_{n, A}}}$, thus collapsing the state on Bob's side to

$$
W_{\varphi}\left|\psi_{k,\left\{j_{n}\right\}}\right\rangle=W_{\varphi}\left(\sum_{n=0}^{N} e_{n} \omega^{k n}\left|b_{n,\left\{j_{n}\right\}}\right\rangle_{B}\right)
$$

with $\left|b_{n,\left\{j_{n}\right\}}\right\rangle_{B}:=\sum_{l_{n}=1}^{d_{n}} \lambda_{n, l_{n}} v^{-j_{n} l_{n}}\left|n, l_{n}\right\rangle_{B}$. Upon knowing the outcome of Alice's measurement, Bob can achieve the minimum cost $\langle c\rangle_{\min }^{j o i n t}$ by performing the optimal phase estimation for the input state $\left|\psi_{k,\left\{j_{n}\right\}}\right\rangle$.

Our result gives a direct way to measure the amount of $\mathrm{RF}$ resource contained in a pure bipartite state. Since we have optimized over all LOCC protocols, the minimum cost $\langle c\rangle_{\min }^{\text {joint }}$ defines a function $F_{c}=-\langle c\rangle_{\min }^{j o i n t}$ on bipartite states $|E\rangle$ which is non-increasing under LOCC operations. Moreover, $F_{c}(|E\rangle\langle E|)$ is also non-increasing under arbitrary operations that commute with local phase shifts 22. In other words, all cost functions define a family of monotone measures $F_{c}$ of bipartite frameness [14, 15, so that the state with greatest frameness is the one with smallest cost. Note that different cost functions induce inequivalent measures, since they correspond to different ways of gauging the quality of a reference frame. E.g., using the cost $c_{v a r}(\varphi)=4 \sin ^{2}(\varphi / 2)$, the state with maximum frameness is the two-mode extension of the optimal clock state [23], namely $\left|E_{\text {var }}\right\rangle=$
$\sum_{n=0}^{N} \sin \left[\frac{\pi(n+1 / 2)}{N+1}\right] / \sqrt{\frac{N+1}{2}}|N-n\rangle_{A}|n\rangle_{B}$, (which has an optimal cost with "Heisenberg bound" scaling $1 / N^{2}$ ). In contrast, using the maximum likelihood cost function $c_{l i k}(\varphi)=-\delta(\varphi)$, the state with maximum frameness is $\left|E_{l i k}\right\rangle \propto \sum_{n=0}^{S}|N-n\rangle|n\rangle$, the two-mode extension of the maximum likelihood state [24]. In all these different cases, our result shows that the performances one can achieve by remote state preparation using an entangled state of $N$ entangled qubits of UI and $N$ classical bits are equivalent to the performances one can achieve by exchanging $N$ qubits of UI and an unlimited amount of SI in an arbitrary amount of rounds of communication between Alice and Bob. Indeed, in the latter scenario the best protocol consists in Alice preparing the $N$ UI qubits in the optimal state and in sending them to Bob 25], thus achieving the same performance of our optimal LOCC protocol.

\section{CONCLUSIONS}

We have shown that perfect teleportation of UI is impossible. In the lack of a shared RF, teleportation is not equivalent to direct quantum communication, not even for a restricted set of input states: teleportation can only transfer the quantum state relative to the sender's reference, but cannot transfer the reference itself. This, however, does not prevent from employing shared entanglement effectively: we have given the optimal protocol for remote preparation of optimal reference states, achieving a quantum metrology enhancement. This protocol can be used to establish an approximate shared RF and then to approximately transfer UI by transmitting only SI.

\section{Acknowledgments}

We thank R.W. Spekkens and S.D. Bartlett for helpful comments. G. C. acknowledges support by the National Basic Research Program of China (973) 2011CBA00300 (2011CBA00302). P.P. acknowledges the FET-Open project COQUIT, grant 233747.
[1] A. Peres and P. F. Scudo, in Quantum Theory: Reconsideration of Foundations, A. Khrennikov, ed. (Vaxjo Univ. Press, Vaxjo, Sweden, 2002).

[2] D. Collins and S. Popescu, quant-ph/0401096 (2004).

[3] S. D. Bartlett, T. Rudolph, and R. W. Spekkens, Rev. Mod. Phys. 79, 555 (2007), pg 603.

[4] C.H. Bennett, G. Brassard, C. Crépeau, R. Jozsa, A. Peres, W.K. Wootters, Phys. Rev. Lett. 70, 1895 (1993).

[5] S. D. Bartlett, H. M. Wiseman, Phys. Rev. Lett. 91, 097903 (2003).
[6] H. M. Wiseman, J. A. Vaccaro, Phys. Rev. Lett. 91, 097902 (2003).

[7] F. Verstraete, J.I. Cirac, Phys. Rev. Lett. 91, 010404 (2003).

[8] S. J. van Enk, J. Mod. Opt. 48, 2049 (2001).

[9] S.D. Bartlett, T. Rudolph, and R.W. Spekkens, Phys. Rev. Lett. 91, 027901 (2003).

[10] R. Jozsa et al., Phys. Rev. Lett. 85, 2010 (2000); E. A. Burt, C. R. Ekstrom, and T. B. Swanson, Phys. Rev. Lett. 87, 129801 (2001). 
[11] J. Preskill, quant-ph/0010098 (2000).

[12] V. Giovannetti, et al. Phys. Rev. A 65, 062319 (2002).

[13] V. Giovannetti, S. Lloyd, L. Maccone, Phys Rev. Lett. 96, 10401 (2006).

[14] G. Gour, R.W. Spekkens, New J. Phys. 10, 033023 (2008).

[15] G. Gour, I. Marvian, R.W. Spekkens, Phys. Rev. A 80, 012307 (2009).

[16] I. Marvian and R.W. Spekkens, arXiv:1104.0018

[17] I. Marvian and R.W. Spekkens, arXiv:1105.1816

[18] Indeed, if the protocol works for any $\varphi$, we can replace $\mathcal{B}_{k}$ with the covariant quantum operation $\mathcal{B}_{k}^{\prime}=$ $\int \mathrm{d} \varphi \mathcal{U}_{\varphi} \mathcal{B}_{k} \mathcal{W}_{\varphi}^{\dagger}$ without affecting the final output state.

[19] Notice that UI-teleportation can be most efficiently achieved without first establishing a common reference frame. Let $\mathcal{H}_{S}=\mathbb{C}^{2}$ and $\mathbf{G}=\left\{\sigma_{\mu}\right\}$ be the "Pauli group" of Pauli matrices. To achieve teleportation with $|E\rangle \propto$ $|0\rangle|0\rangle+|1\rangle|1\rangle$, Alice measures her qubit and her half of the entangled state on the Bell basis $\left|E_{\mu}\right\rangle=\left(\sigma_{\mu} \otimes \mathbb{1}\right)|E\rangle$, and Bob implements the correction $\sigma_{\mu}$.

[20] A. S. Holevo, Probabilistic and Statistical Aspects of Quantum Theory, (North-Holland, Amsterdam, 1982).

[21] G. Chiribella, G. M. D'Ariano, and M. F. Sacchi, Phys. Rev. A 72, 042338 (2005).

[22] Indeed, if a channel $\mathcal{C}$ satisfies $\mathcal{C} \circ\left(\mathcal{I}_{A} \otimes \mathcal{W}_{\varphi}\right)=\left(\mathcal{I}_{A} \otimes\right.$ $\left.\mathcal{W}_{\varphi}\right) \circ \mathcal{C}$, we can apply $\mathcal{C}$ in the Heisenberg picture on the POVM $M_{\hat{\varphi}}^{(\varphi)}$, thus obtaining a new POVM $\widetilde{M}_{\hat{\varphi}}^{(\varphi)}=$ $\left(\mathcal{I}_{A} \otimes \mathcal{W}_{\varphi}\right)(\widetilde{\Xi})$, with $\widetilde{\Xi}=\mathcal{C}^{\dagger}(\Xi)$, which is still of the correct form, whence $F_{c}\left(\mathcal{C}(|E\rangle\langle E|) \leq F_{c}(|E\rangle\langle E|)\right.$.

[23] V. Bužek, R. Derka, and S. Massar, Phys. Rev. Lett. 82, 2207 (1999).

[24] G. Chiribella, G. M. D'Ariano, P. Perinotti, and M. F. Sacchi, Phys. Rev. A 70, 062105 (2004).

[25] G. Chiribella, G. M. D'Ariano, and P. Perinotti, AIP Conf. Proc. 1110, 47 (2009). 